\title{
Brain specific proteins in posthaemorrhagic ventricular dilatation
}

\author{
A Whitelaw, L Rosengren, M Blennow
}

\begin{abstract}
Median neurofilament and glial fibrillary acidic protein concentrations in the cerebrospinal fluid of 18 infants with posthaemorrhagic ventricular dilatation were 20-200 times higher than control values. S-100 protein in cerebrospinal fluid was four times higher than control values. Glial fibrillary acidic protein concentrations correlated with death or disability and with parenchymal lesions but not with shunt dependence.
\end{abstract}

(Arch Dis Child Fetal Neonatal Ed 2001;84:F90-F91)

Keywords: cerebrospinal fluid; glial fibrillary acidic protein; posthaemorrhagic ventricular dilatation; brain; neurodevelopment

Division of Child

Health, University of Bristol, Bristol, UK A Whitelaw

Department of Neurology,

Sahlgrenska Hospital, Gothenburg, Sweden

L Rosengren

Department of Paediatrics, Huddinge Hospital, Huddinge, Sweden

M Blennow

Correspondence to:

Professor Whitelaw, Division

of Child Health, University

of Bristol Medical School,

Southmead Hospital, Bristol

BS10 5NB, UK

email: andrew.whitelaw@

bristol.ac.uk

Accepted 21 January 2000

Preterm infants with ventricular dilatation following intraventricular haemorrhage (IVH) often have cerebral palsy and cognitive disabilities, even in the absence of parenchymal lesions visible by cranial ultrasound. ${ }^{1}$ Neuropathological and experimental studies indicate that these neurological impairments result from injury to periventricular white matter. Ultrasound is recognised to detect less than $50 \%$ of infants with white matter injury shown at autopsy. White matter injury has begun to be evaluated by brain specific proteins in the cerebrospinal fluid (CSF).

The light subunit of the neurofilament triplet protein (NFL) is the essential component of the neurofilament core in axons and can be used as a marker of axonal degeneration. ${ }^{2}$ Glial fibrillary acid protein (GFAP) is the major structural protein of fibrillary astrocytes, and

Table 1 Median (ranges) for cerebrospinal fluid concentrations of brain specific proteins

\begin{tabular}{|c|c|c|c|}
\hline & PHVD - parenchymal lesion & PHVD + parenchymal lesion & $p$ Value \\
\hline $\mathrm{n}$ & 7 & 11 & \\
\hline NFL (ng/l) & $2563(713-107000)$ & $36446(1846-168000)$ & 0.02 \\
\hline GFAP (ng/l) & $24849(6808-67326)$ & $196500(15041-1100000)$ & 0.002 \\
\hline \multirow[t]{2}{*}{ S-100 ( $\mu \mathrm{g} / 1)$} & $3.56(2.19-9.73)$ & $9.85(2.19-36)$ & 0.036 \\
\hline & $\begin{array}{l}\text { PHVD } \\
\text { normal at } 12 \text { months }\end{array}$ & $\begin{array}{l}\text { PHVD } \\
\text { disability at } 12 \text { months }\end{array}$ & $p$ Value \\
\hline $\mathrm{n}$ & 7 & 11 & \\
\hline NFL (ng/l) & $2563(713-107000)$ & $38092(1846-168000)$ & 0.0154 \\
\hline GFAP (ng/l) & $24849(6808-67236)$ & $196500(15041-1100000)$ & 0.002 \\
\hline \multirow[t]{2}{*}{$\mathrm{S}-100(\mu \mathrm{g} / \mathrm{l})$} & $3.56(2.19-9.73)$ & $9.85(2.19-36)$ & 0.036 \\
\hline & PHVD no shunt & PHVD shunt or dead & $p$ Value \\
\hline $\mathrm{n}$ & 6 & 12 & \\
\hline NFL (ng/l) & $22093(1448-168000)$ & $7622(713-88168)$ & 0.437 \\
\hline GFAP (ng/l) & $58797(10354-154000)$ & $149250(6808-1100000)$ & 0.291 \\
\hline$S-100(\mu \mathrm{g} / 1)$ & $4.78(2.19-19)$ & $8.76(2.19-36)$ & 0.30 \\
\hline
\end{tabular}

Normal preterm control: NFL, median (range) $125(<125-138)$ ng/l $(\mathrm{n}=4)$; GFAP, mean (SD) $106(15-362) \mathrm{ng} / \mathrm{l}(\mathrm{n}=17)$; S-100, median (range) $0.82(0.39-1.09) \mu \mathrm{g} / \mathrm{l}(\mathrm{n}=4)$. p Values obtained using the Mann-Whitney U test.

PHVD, posthaemorrhagic ventricular dilatation; NFL, neurofilament triplet protein; GFAP, glial fibrillary acid protein. its presence in CSF is related to astroglial damage. ${ }^{3}$ GFAP in CSF of preterm infants is increased fivefold in preterm infants with an abnormal neonatal course or abnormal neurological signs. ${ }^{4}$

$\mathrm{S}-100$ is an acidic calcium binding protein produced in both protoplasmic and fibrillary astrocytes. ${ }^{5}$ These proteins are not normally synthesised outside the central nervous system and are not present in erythrocytes, leucocytes, or platelets.

The objects of this study were to determine whether the brain specific proteins GFAP, NFL, and S-100 in the CSF of preterm infants with posthaemorrhagic ventricular dilatation (PHVD) differed from control preterm infants and were related to neurodevelopmental outcome, the presence of parenchymal brain lesions on ultrasound, and the need for ventriculoperitoneal shunting.

\section{Methods}

CSF surplus to diagnostic requirements was available after clinically indicated ventricular puncture in 18 preterm infants of mean (SD) birth weight 1287 (490) g with PHVD defined as IVH followed by progressive enlargement of ventricular width reaching $4 \mathrm{~mm}$ over the 97 th centile for each lateral ventricle. ${ }^{1}$

Control values for GFAP were previously obtained in our laboratory from 17 preterm infants (mean (SD) birth weight 1242 (360) g). ${ }^{4}$ Control values for NFL and S-100 were obtained from CSF samples surplus to requirements from four preterm infants with birth weights 939-1520 g. All the control infants were lumbar punctured on clinical indications to exclude infection but were retrospectively found to have no evidence of infection or IVH and had normal outcome on follow up.

CSF samples were centrifuged, and the supernatants frozen and transported to the laboratory. NFL and GFAP concentrations were measured using sandwich enzyme linked immunoassays. ${ }^{26} \mathrm{~S}-100$ protein was measured using a commercially available luminometric immunoassay (Sangtec Medical, Bromma, Sweden).

\section{Results}

Table 1 shows that CSF concentrations of GFAP, NFL, and S-100 from infants with PHVD were higher in those with parenchymal brain lesions and those who died or were disabled at 12 months (Ruth Griffiths developmental quotient $<70$ ). The differences for GFAP were highly significant, but those for NFL and S-100 were of borderline significance. Infants with PHVD but no parenchymal 
lesions and normal development had a median CSF concentration of GFAP that was over 200 times that in the normal control infants, with no overlap. In the case of NFL, the factor was almost 20 times that in the normal control infants. Median S-100 concentration in the PHVD group was over four times the median of the control values with no overlap. There was no significant difference in NFL, GFAP, and S-100 concentrations between the group who survived without shunting and the group who required shunt surgery or died with progressive hydrocephalus.

\section{Discussion}

The sampling technique in the infants with PHVD involved ventricular tap in all but one case. The one infant from whom samples were taken by ventricular reservoir had brain specific protein concentrations comparable with those of the other infants with PHVD. We cannot rule out the possibility that ventricular puncture introduced brain proteins into the CSF, although such a process would not account for the further increases in brain proteins in infants with parenchymal lesions and infants with later disability. We have not had the opportunity to compare simultaneous ventricular and lumbar samples so we cannot rule out the possibility that ventricular concentrations are consistently higher than lumbar concentrations.

The extremely high GFAP concentrations in the 18 infants with PHVD are comparable with those observed after acute parenchymal destruction in adult brain, such as herpes encephalitis and cerebral infarctions, but much higher than those observed in chronic neurodegenerative disorders with concurrent astrogliosis. ${ }^{36}$ The NFL levels in the present study are much higher than those observed in CSF from patients with chronic neurodegenerative disorders but closer to those observed after large cerebral infarctions and herpes encephalitis. ${ }^{2}$ The CSF concentrations of S-100 in the infants with PHVD were comparable with those in adults with acute brain tissue damage. ${ }^{3}$

The full extent of periventricular white matter injury in the preterm infant cannot be assessed by either repeated cranial ultrasound examinations or neurodevelopmental assessment at 12 months. Grossly elevated concentrations of brain specific proteins, especially GFAP, in the CSF provide further evidence of periventricular white matter damage in infants with PHVD.

This study was supported by the Cornell Foundation.

1 Ventriculomegaly Trial Group. Randomised trial of early tapping in neonatal posthaemorrhagic ventricular dilatation. Arch Dis Child Fetal Neonatal Ed 1994;65:F3-10.

2 Rosengren LE, Karlsson JE, Karlsson JO, Persson LI, Wikkelsö. Patients with amyotrophic lateral sclerosis and other neurodegenerative diseases have increased levels of neurofilament protein in CSF. 7 Neurochem 1996;67:2013-18.

3 Aurell A, Rosengren LE, Karlsson B, Olsson JE, Zbornikova V, Haglid KG. Determination of S-100 and glial fibrillary acidic protein concentrations in cerebrospinal fluid after brain infarction. Stroke 1991;22:1254-8.

4 Blennow M, Rosengren L, Jonsson S, et al. Glial fibrillary acid protein is increased in the cerebrospinal fluid of preterm infants with abnormal neurological findings. Acta Paediatr 1996;85:485-9.

5 Persson L, Hårdemark HG, Gustafsson J, et al. S-100 protein and neuron-specific enolase in cerebrospinal and serum: markers of cell damage in human central nervous system. Stroke 1987;18:911-18.

6 Rosengren LE, Wikkelso C, Hagberg L. A sensitive ELISA for glial fibrillary acidic protein: applications in CSF of adults. F Neurosci Methods 1994;51:197-204. 